\title{
Metal Object Detection in a Wireless Power Transfer System Based on Double-Layer Symmetric Sensing Coils
}

\author{
Yawen Lin, Lijuan Xiang, Jindong Tian and Yong Tian* \\ College of Physics and Optoelectronic Engineering, Shenzhen University, Shenzhen, Guangdong, 518060, China
}

\begin{abstract}
The intrusion of a metal object into a wireless charging system could cause safety issues, such as temperature rise and even combustion. This paper proposes a double-layer symmetric sensing coil design for metal object detection (MOD) of wireless electric vehicle charging to prevent the detection blind area. First, the magnetic effect and the eddy current effect of a metal object in high-frequency magnetic fields are analyzed. Second, the principle of the proposed double-layer symmetric sensing coils is detailed. Finally, the finite element simulation is implemented in order to test the performance of the proposed sensing coil design. The simulation results show that the proposed double-layer symmetric sensing coils can effectively detect the presence and position of the metal object simultaneously.
\end{abstract}

\section{Introduction}

Wireless power transfer (WPT) has the advantages of safety, highly efficiency and reliability, thus it has been widely used in electric vehicles, household appliances and other commercial fields [1]-[2]. However, due to the special structure of a wireless charging system, a large air gap filled with high power and high frequency magnetic fields exists between the transmitting (Tx) coil and the receiving $(\mathrm{Rx})$ coil. When a metal object such as an iron piece, a coin and a key intrudes into the charging area, eddy current will be generated inside the metal object. Especially, if a large number of eddy current are generated in a high-resistance metal object, the high temperature caused by ohmic losses will eventually lead to safety concerns such as combustion. To avoid such a situation, some metal object detection (MOD) technologies have been developed. A method for detecting system parameters is proposed in [3]-[4], which is based on measuring the voltage, current, power, phase and frequency of the resonator, or the quality factor of the $\mathrm{Tx} / \mathrm{Rx}$ coil with and without metal objects. However, the Tx coil for wireless electric vehicle charging is much larger than a general metal object, so it is difficult to detect the slight change of system parameters caused by the intrusion of a small metal object. In addition, the system parameters are not only affected by metal objects, but also by other factors such as air gap change and misalignment between the Tx coil and the Rx coil. A method by comparing power loss with and without metal objects has been applied to Qi standard method [5] because of its simple operation and low cost. However, considering that the power loss caused by a metal object is usually only a few watts, which is a small part of the total power transmitted by the WPT system. Therefore, this method is difficult to be applied for high power transmission. Metal objects and organisms can be also detected by using radar, thermal imager or video camera [6]-[8]. However, these methods are relatively costly, and are easily affected by the environment, such as dust and sewage. A basic magnetic field orientation method is to arrange several additional sensing coils between the Tx coil and the Rx coil, in which the magnetic field distribution is different with and without metal objects [9]-[10]. Among these solutions, methods based on magnetic field distribution are currently considered to be the best choice for real applications, because they are easy to be implemented and have simple and effective structures. Nevertheless, they usually suffer from the dilemma of detection blind area.

In the paper, an effective and reliable double-layer symmetric sensing coil structure is proposed to prevent the blind area of metal object detection, which uses the voltage difference of the symmetric sensing coil to detect the presence of a metal object. Theoretical analysis and simulation validation will be introduced in the following chapters to verify the effectiveness and feasibility of the proposed double-layer symmetric sensing coils.

\section{The influence of a metal object on magnetic fields}

\subsection{Metal magnetic effect}

In the process of wireless charging, various foreign objects may intrude into the charging area. Among them, the metal object is harmful and likely to affect charging efficiency and safety. Metal objects intruding into the wireless charging area will cause a series of electromagnetic

*Corresponding author's e-mail: ytian@szu.edu.cn 
phenomena because of the high-frequency electromagnetic fields. In particular, metal objects with high permeability will change the magnetic field distribution. As an example, Figure 1 shows the mechanical dimensions of a typical Tx coil recommended by SAE J2954TMNov2017, and an iron sheet with a size of $50 * 50 * 2 \mathrm{~mm} 3$ is located on the surface of the Tx coil. The simulation results of magnetic induction intensity distribution in electromagnetic coupling region are shown in Figure 2. As shown in Figure 2(b), when the iron sheet is placed in the charging area, the magnetic induction intensity of the location of the iron sheet and surrounding area changes significantly. In order to visually observe the change of the transmitted magnetic field before and after placing the iron sheet, magnetic induction intensities with $\mathrm{B}(\mathrm{MO})$ and without $\mathrm{B}(\mathrm{WO})$ an iron sheet along a horizontal line parallel to the $\mathrm{X}$-axis through the center point $(0 \mathrm{~mm},-100 \mathrm{~mm}, 12 \mathrm{~mm})$ are shown in Figure 2(c).

It can be seen from Figure 2(c) that original magnetic fields generated by the Tx coil are basically symmetrical. After the intrusion of an iron sheet, the magnetic induction intensity B around the iron sheet changes obviously, and it changes the most at the position $X=25 \mathrm{~mm}$, which decreases about $0.484 \mathrm{mT}$. Therefore, it is proved that the metal object will change the distribution of the magnetic induction intensity of the Tx coil.

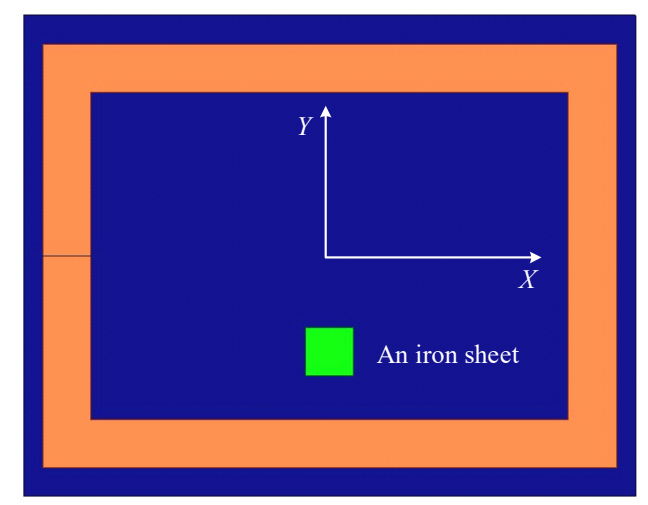

Figure 1. Schematic diagram of the Tx coil structure.

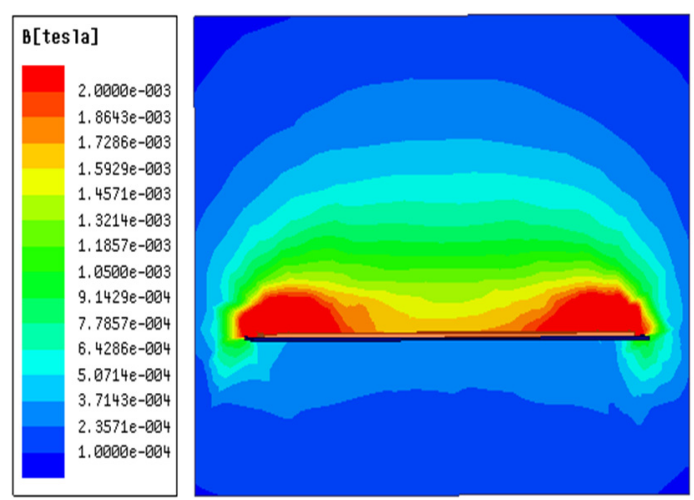

(a)

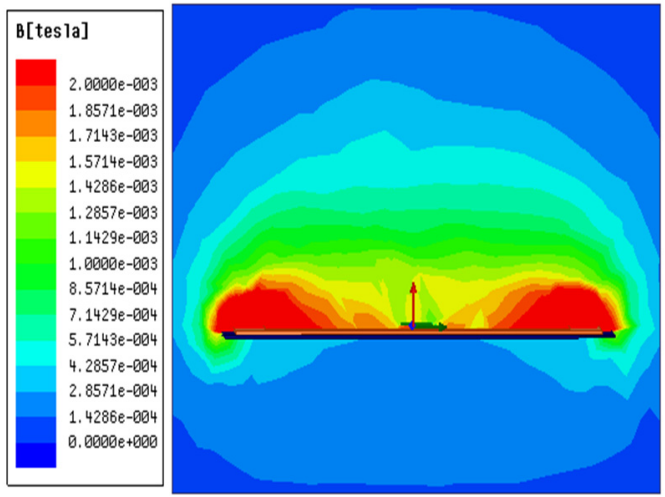

(b)

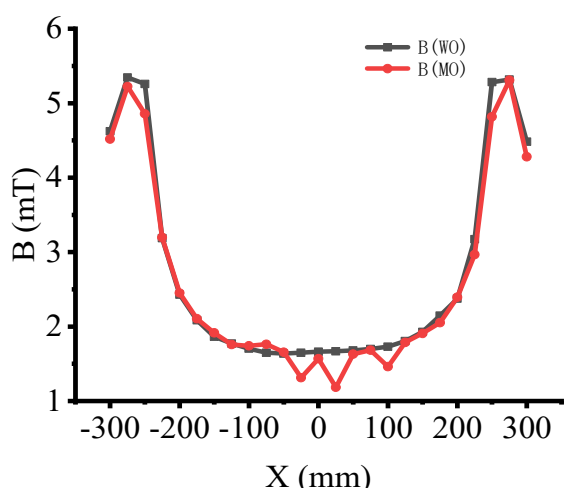

(c)

Figure 2. Distribution of magnetic induction intensities with and without an iron sheet.

\subsection{Eddy current effect}

When a metal object is located in alternating current (AC) magnetic fields, the eddy current will be generated in the metal object, causing changes in inductance, resistance and quality factor of the Tx coil. By ignoring the change of the Rx coil, and only considering the eddy current effects of the Tx coil and the metal object, impedance characteristics of the Tx coil before and after placing a metal object are analyzed here.

A metal object locating in magnetic fields can be equivalent to a series circuit of a resistor and an inductor, and mutual inductance exists between the eddy current inductance and the Tx coil. The equivalent circuit is shown in Figure 3, where AC is the equivalent source, $R_{2}$ and $L_{2}$ represent resistance and inductance of the metal object, respectively, and $M$ is the mutual inductance between the Tx coil and the metal object.

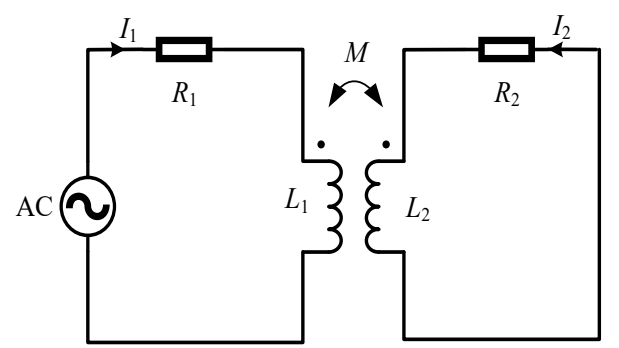

Figure 3. Schematic of the eddy current equivalent circuit.

When there is no metal objects on the Tx coil, impedance of the Tx coil is 


$$
Z=R_{1}+j \omega L_{1}
$$

where $\omega$ is the system operating frequency.

After the intrusion of a metal object, based on Figure 3 , we can get

$$
\left\{\begin{array}{l}
R_{1} \dot{I}_{1}+j \omega L_{1} \dot{I}_{1}-j \omega M \dot{I}_{2}=\dot{U} \\
R_{2} \dot{I}_{2}+j \omega L_{2} \dot{I}_{2}-j \omega M \dot{I}_{1}=0
\end{array}\right.
$$

Then, the equivalent impedance of the Tx coil can be deduced as

$$
Z^{\prime}=\frac{\dot{U}_{1}}{\dot{I}_{1}}=R_{1}+\frac{R_{2} \omega^{2} M^{2}}{R_{2}^{2}+\omega^{2} L_{2}^{2}}+j \omega\left(\mathrm{L}_{1}-\frac{\omega^{2} M^{2} L_{2}}{R_{2}^{2}+\omega^{2} L_{2}^{2}}\right)
$$

Seen from the Tx coil, the equivalent resistance and equivalent inductance can be rewritten as

$$
\begin{aligned}
& R=R_{1}+\frac{R_{2} \omega^{2} M^{2}}{R_{2}^{2}+\omega^{2} L_{2}^{2}} \\
& L=L_{1}-\frac{\omega^{2} M^{2} L_{2}}{R_{2}^{2}+\omega^{2} L_{2}^{2}}
\end{aligned}
$$

By comparing Equation (1) with Equations (4) and (5), it is clear that the equivalent resistance increases, while the equivalent inductance decreases due to the intrusion of a metal object.

\section{Principle of the proposed double-layer symmetric sensing coils}

The Tx coil is energized with high-frequency AC current, the magnetic flux $\phi(t)$ will be generated in the sensing coil. According to Faraday's law, the induced voltage in the sensing coil is equal to the derivative of magnetic flux $\phi(t)$, and it can be formulated as

$$
V_{k}=V_{k \mathrm{a}}=V_{k \mathrm{~b}}=\frac{\mathrm{d} \phi(t)}{\mathrm{d} t}=\frac{\mathrm{d} B(t) \times S}{\mathrm{~d} t}=\omega M_{k} I_{1}
$$

where $S$ is the area of the sensing coil.

Based on the symmetrical distribution of magnetic fields in horizontal and vertical directions, symmetric sensing coils are proposed in the paper to prevent detection blind area for metal objects. As shown in Figure 4 , the proposed symmetric sensing coils include a plurality of rectangular sensing coils, covering the whole area of the Tx coil, and are mainly divided into the first layer of the vertical sensing coils (i.e., $1 \mathrm{a}, 2 \mathrm{a}, \ldots, 12 \mathrm{a}$ and $1 \mathrm{~b}, 2 \mathrm{~b}, \ldots$, $12 \mathrm{~b})$ and the second layer of the horizontal sensing coils (i.e., 1c,2c,2d,1d). The vertical sensing coils are divided into 12 channels, which are symmetrically distributed about the center X-axis of the Tx coil, and each channel includes two symmetric sensing coils. For example, channel 1 includes coil $1 \mathrm{a}$ and $1 \mathrm{~b}$, and channel 2 includes coil $2 \mathrm{a}$ and $2 \mathrm{~b}$, up to channel $\mathrm{n}$, including coil $n \mathrm{a}$ and $n \mathrm{~b}$. The horizontal induction detection coils are divided into two channels, which are symmetrically distributed about the center Y-axis of the Tx coil. Channel 1 includes coil 1c and $1 \mathrm{~d}$, and channel 2 includes coil $2 \mathrm{c}$ and $2 \mathrm{~d}$.

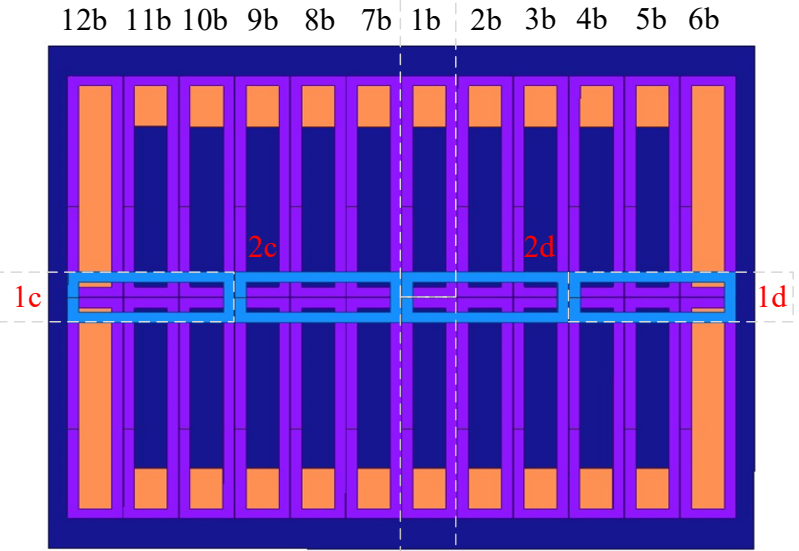

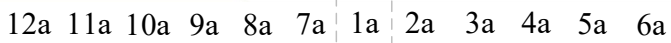

Figure 4. The configuration of the proposed symmetric sensing coils.

In wireless electric vehicle charging, the two sensing coils of one channel generate the same induced voltage. When there is no metal objects on the sensing coil, the magnetic flux $\phi(t)$ flow through the two sensing coils of one channel in the opposite direction, and the magnetic flux $\phi(t)$ will cancel each other out. Consequently, the induced voltage difference of the two sensing coils of one channel is defined as

$$
\Delta V_{k}=V_{k \mathrm{a}}-V_{k \mathrm{~b}}=0
$$

If a metal object intrudes into and locates on one sensing coil of a coil channel, the eddy current induced in the metal object will change the distribution of the magnetic field, thus causing a deviation $\Delta V_{k}$ between the induced voltages $V_{k \mathrm{a}}$ and $V_{k \mathrm{~b}}$ as shown in Equation (8), which can be used to determine the presence of a metal object.

$$
\Delta V_{k}=V_{k \mathrm{a}}-V_{k \mathrm{~b}} \neq 0
$$

According to Equation (6), the induced voltage is proportional to the mutual inductance, so the presence of a metal object also can be detected by the mutual inductance difference

$$
\Delta M_{k}=|M(k \mathrm{a}, \mathrm{P})|-|M(k \mathrm{~b}, \mathrm{P})|
$$

As shown in Figure 5, when there is no metal objects, it is obvious that the mutual inductance differences $\Delta M_{k}$ between the two sensing coils in one channel on the lower side are positive and the absolute values are the same as the relevant ones on the upper side, indicating that the difference $\Delta M_{k}$ is zero. In order to verify the detection effect of the proposed symmetric sensing coils, a coin with a radius of $15 \mathrm{~mm}$ is applied as the test object. As an example, $\Delta M_{1}$ is presented in Figure 6. It can be seen that $\Delta M_{1}$ increases with the increase of the coin number. In particular, as the coin number increases from one to six, $\Delta M_{1}$ increases from $173.59 \mathrm{nH}$ to $281.86 \mathrm{nH}$. 


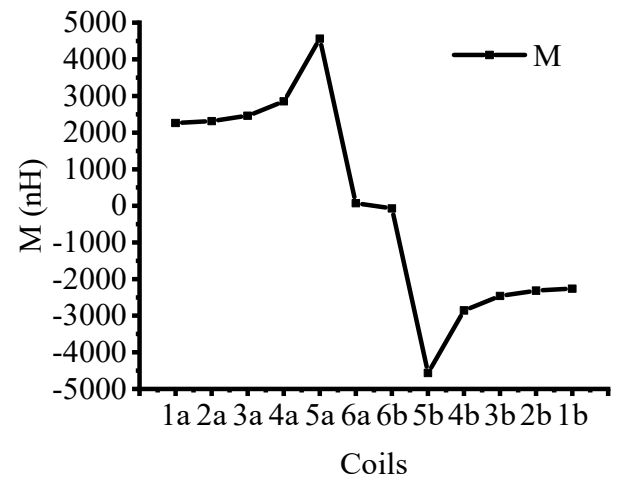

Figure 5. Mutual inductance between the symmetrical sensing coils and the Tx coil in vertical direction.

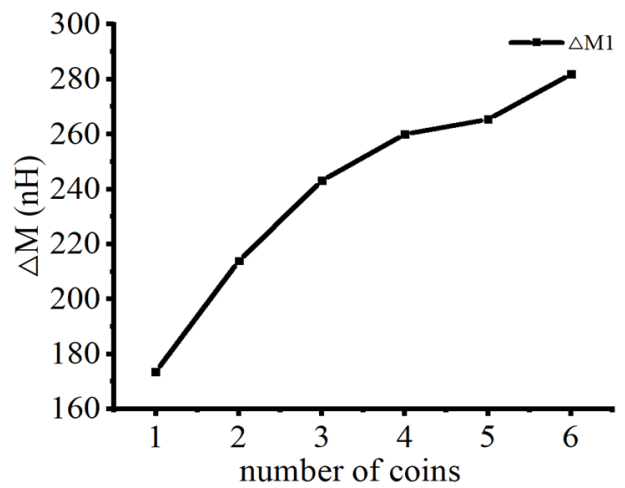

Figure 6. Variation of $\Delta M_{1}$ with the increase in coin number.

\section{Simulation validation and discussion}

\subsection{MOD results in vertical direction}

In this section, the finite element simulation (FEM) with Ansys Maxwell16.0 software is carried to verify the effectiveness of the proposed symmetric sensing coils. Since magnetic fields on the left and right sides of the Tx coil are symmetrically distributed, only the right half of symmetric sensing coils (i.e., 1a, 2a, ..., 6a and 1b, 2b, ..., 6b) of the first layer is used for feasibility study in order to simplify the simulation. Parameters of the Tx coil and the sensing coil are listed in Table 1 . A coin with the size of $15^{*} 2 \mathrm{~mm}^{2}$ is placed on the top of the sensing coil, and it is initially located at $(0 \mathrm{~mm},-200 \mathrm{~mm})$. When the coin moves along the $\mathrm{X}$-axis with a step size of $50 \mathrm{~mm}$ and the moving distance ranges from $0 \mathrm{~mm}$ to $275 \mathrm{~mm}, \Delta M_{k}$ in Equation (9) is evaluated as shown in Figure 7.

Table 1. Simulation parameters

\begin{tabular}{cc}
\hline Parameters & Values \\
\hline Ferrite core size & $640 * 500 * 3 \mathrm{~mm}^{3}$ \\
Primary Litz coil size & $600 * 440 * 5 \mathrm{~mm}^{3}$ (outer) \\
Primary coil turns & $10 * 340 * 5 \mathrm{~mm}^{3}$ (inner) \\
Primary coil current & $30 \mathrm{~A}$ \\
Vertical sensing detection \\
coil size
\end{tabular}

\begin{tabular}{cc}
\hline Horizontal sensing & $30 * 200 * 2 \mathrm{~mm}^{3}$ (inner) \\
detection coil size & $151 * 50 * 2 \mathrm{~mm}^{3}$ (outer) \\
& $131 * 30 * 2 \mathrm{~mm}^{3}$ (inner) \\
Sensing coil turns & 5 \\
\hline
\end{tabular}

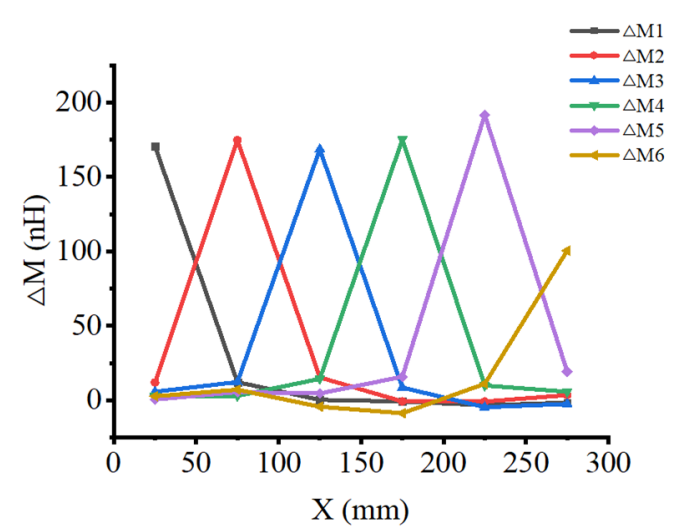

(a)

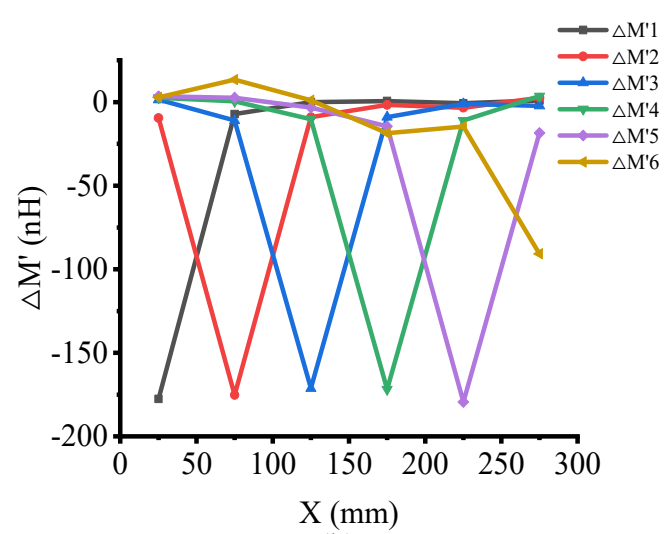

(b)

Figure 7. Change of $\Delta M_{k}$ with the difference position of the coin on (a) lower half and (b) upper half of the Tx coil.

As shown in Figure 7, all mutual inductance differences $\Delta M_{k}$ change with the variation of coin position. When the coin is placed on top of the sensing coil from 1a to $6 \mathrm{a}$, the corresponding $\Delta M_{k}$ reaches its peak. For example, according to the blue curve that presents the change of the mutual inductance difference $\Delta M_{3}$ between the sensing coil $3 \mathrm{a}$ and $3 \mathrm{~b}$ with the change of coin position, it can be seen that when the coin is at the position of $X=125 \mathrm{~mm}$ (i.e., on the top of the sensing coil $3 \mathrm{a}$ ), $\Delta M_{3}$ gets the maximum value of $168.6 \mathrm{nH}$, which is greatly higher than the other $\Delta M_{k}$ values. Similarly, as the coin moves on the sensing coils from $1 \mathrm{~b}$ to $6 \mathrm{~b}$, the mutual inductance differences $\Delta M_{k}^{\prime}$ exhibit the same change rule, as shown in Figure 7(b). Additionally, because of the magnetic field direction in the two sensing coils of a channel is opposite, the position of the metal object can be determined by the sign of the mutual inductance difference. For example, a positive value of $168.6 \mathrm{nH}$ indicates that the coin is on coil $3 \mathrm{a}$, and a negative value of $-171.2 \mathrm{nH}$ indicates that the coin is located on coil $3 b$. 


\subsection{MOD results in horizontal direction}

If only the symmetric sensing coils in vertical direction are considered, detection blind areas are likely to exist on the Tx coil. For example, as shown in Figure 8, if a coin is located at $(125 \mathrm{~mm}, 0 \mathrm{~mm})$, covering the same area of the sensing coils $3 a$ and $3 b$, the mutual inductance difference $\Delta M_{3}$ is extremely approximate to zero, thus the system is not able to detect the presence of the coin (detection blind area). Aiming to this problem, this study adds an extra second layer of symmetric sensing coils in horizontal direction, namely $1 \mathrm{c}, 2 \mathrm{c}, 2 \mathrm{~d}$ and $1 \mathrm{~d}$, which are symmetrically located at the bottom of the first layer of sensing coils. To distinguish from the first layer of sensing coils, the differences of mutual inductance $\Delta M_{k}^{2}$ of the second layer of sensing coils are marked with an upper angle.

Figure 9 presents the differences of mutual inductance $\Delta M_{1}^{\prime}$ and $\Delta M_{2}^{\prime}$ as the coin moves from $-275 \mathrm{~mm}$ to 275 $\mathrm{mm}$ along the $\mathrm{X}$-axis with a step of $50 \mathrm{~mm}$. It can be clearly seen that $\left|\Delta M_{1}^{\prime}\right|$ is greater than $\left|\Delta M_{2}^{\prime}\right|$ when the coin is located in the range of [-275 mm, $-150 \mathrm{~mm}]$ and [150 mm, $275 \mathrm{~mm}]$, which indicates that the coin is located on the sensing coil 1c or 1d. However, $\left|\Delta M_{2}^{\prime}\right|$ is greater than $\left|\Delta M_{1}^{\prime}\right|$ when the coin is located in the range of [-150 mm, $150 \mathrm{~mm}]$, meaning that the coin is located on the sensing coil $2 \mathrm{c}$ or $2 \mathrm{~d}$. Consequently, the detection blind area is overcome by the added a second layer of sensing coils.

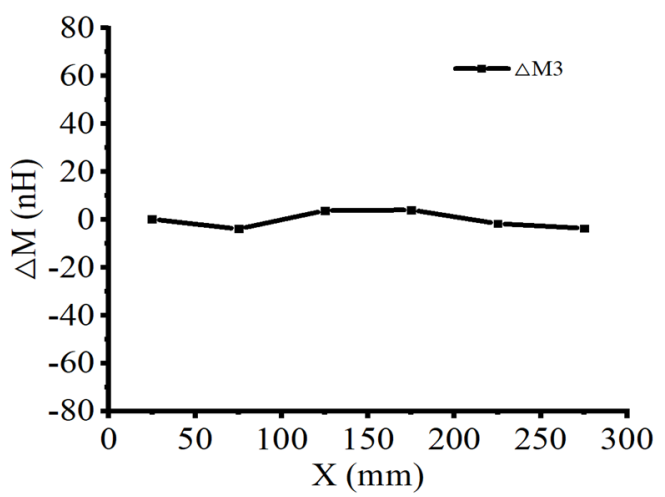

Figure 8. Mutual inductance in the blind area.

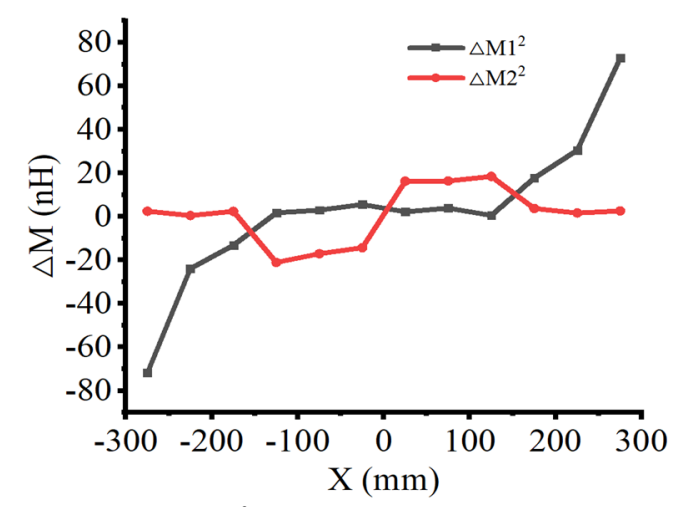

Figure 9. $\Delta M_{k}^{2}$ of the horizontal sensing coils.
In addition, according to the 15 types of test bodies recommended by IEC 61980-3 Table 309, this paper selects the steel sheet with the size of $20 * 20 * 2 \mathrm{~mm}^{3}$ and the screw with the radius of $8 \mathrm{~mm}$ and the high of $20 \mathrm{~mm}$ to further evaluate the proposed symmetric sensing coils. Because all the symmetric sensing coils have the same change rules, here only the change of mutual inductance difference in channel 5 is observed. Figure 10(a) and Figure 10(b) show the change of mutual inductance differences $\Delta M_{5}$ and $\Delta M_{5}^{\prime}$ with the change of metal object position, respectively. It is indicated that $\Delta M_{5}$ gets the peak value when the metal objects are located on the symmetric sensing coil $5 \mathrm{a}$ or $5 \mathrm{~b}$. It is similar to the previous results in Figure 7. Thus, it is proved that the proposed symmetric sensing coils can successfully detect different metal objects.

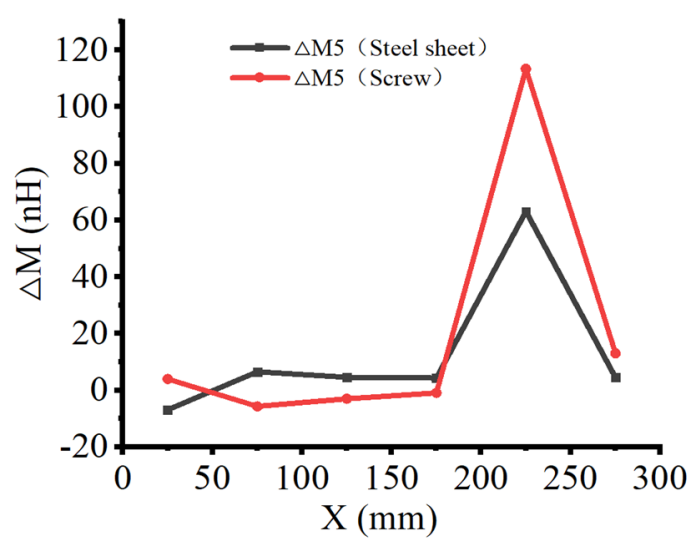

(a)

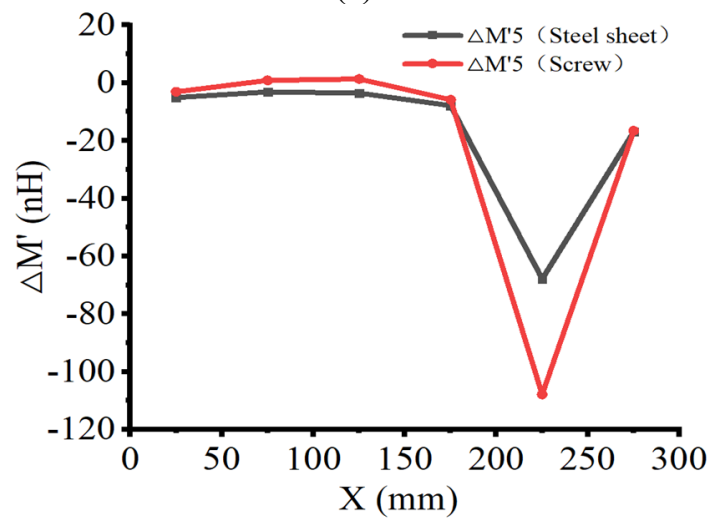

(b)

Figure 10. Mutual inductance difference in channel 5 for detecting different metal objects.

\section{Summary}

In the paper, double-layer symmetric sensing coils are designed to detect the intrusion of a metal object on the Tx coil of the electric vehicle wireless charging system. The influence of the metal object on magnetic fields generated by the Tx coil is analyzed from the respects of magnetic effect and eddy current effect. Principle of the proposed symmetric coils is introduced in detail. With the proposed symmetric sensing coils, metal objects are detected by the symmetric sensing coils in horizontal and vertical directions simultaneously in order to eliminate the 
detection blind area. In particular, the presence of a metal object is determined by the voltage difference or mutual inductance difference between the two symmetrical coils in a channel, and the position is determined by the sign of mutual inductance difference. Simulation results reveal that the proposed double-layer symmetric sensing coils are effective to detect different metal objects and can well prevent the detection blind area.

\section{Acknowledgments}

This work was supported by the Science and Technology Plan Project of Shenzhen (No. JCYJ20170412110241478).

\section{References:}

1. Choi, S. Y., Gu, B. W., Jeong, S. Y. and Rim, C. T. (2015) Advances in Wireless Power Transfer Systems for Roadway-Powered Electric Vehicles. IEEE Journal of Emerging and Selected Topics in Power Electronics., 3: 18-36.

2. Li, S. and Mi, C. C. (2015) Wireless Power Transfer for Electric Vehicle Applications. IEEE Journal of Emerging and Selected Topics in Power Electronics., 3: 4-17.

3. Fukuda, S., Nakano, H., Murayama, Y., Murakami, T., Kozakai, O. and Fujimaki, K. (2012) A Novel Metal Detector Using the Quality Factor of the Secondary Coil for Wireless Power Transfer Systems. In: IEEE MTT-S International Microwave Workshop Series on Innovative Wireless Power Transmission: Technologies, Systems, and Applications. Kyoto. pp. 241-244.

4. Low, Z. N., Casanova, J. J., Maier, P. H., Taylor, J. A., Chinga, R. A. and Lin, J. (2010) Method of Load/Fault Detection for Loosely Coupled Planar Wireless Power Transfer System with Power Delivery Tracking. IEEE Transactions on Industrial Electronics., 57: 1478-1486.

5. Kuyvenhoven, N., Dean, C., Melton, J., Schwannecke, J. and Umenei, A. E. (2011) Development of a Foreign Object Detection and Analysis Method for Wireless Power Systems. In: IEEE Symposium on Product Compliance Engineering Proceedings. San Diego, CA. pp. 1-6.

6. Xu, Q.Y., Ning, H.S. and Chen W.S. (2009) VideoBased Foreign Object Debris Detection. In: IEEE International Workshop on Imaging Systems and Techniques. Shenzhen. pp. 119-122.

7. Lee, K. and Cho, D. (2013) Diversity Analysis of Multiple Transmitters in Wireless Power Transfer System. IEEE Transactions on Magnetics., 49: 29462952.

8. Kohmura, A., Futatsumori, S., Yonemoto, N. and Okada, K. (2013) Optical Fiber Connected Millimeter-Wave Radar for FOD Detection On Runway. In: European Radar Conference. Nuremberg. pp. 41-44.
9. Jeong, S. Y., Kwak, H. G., Jang, G. C., Choi, S. Y. and Rim, C. T. (2018) Dual-Purpose Nonoverlapping Coil Sets as Metal Object and Vehicle Position Detections for Wireless Stationary EV Chargers. IEEE Transactions on Power Electronics., 33: 73877397.

10. Thai, V. X., Park, J. H., Jeong, S. Y., Rim, C. T. and Kim, Y. (2020) Equivalent-Circuit-Based Design of Symmetric Sensing Coil for Self-Inductance-Based Metal Object Detection. IEEE ACCESS., 8: 9419094203. 